Abstract

\title{
Comparison of Radical Scavenging and Cytotoxic Activities of Well-Known Non-Enzymatic Antioxidants ${ }^{+}$
}

\author{
Suna Sabuncuoğlu *, Ayşe Zeynep Ünal and Gözde Girgin * \\ Toxicology Department, Faculty of Pharmacy, Hacettepe University, Ankara 06000, Turkey; \\ a.zeynep.u@gmail.com \\ * Correspondence: suna@hacettepe.edu.tr (S.S.); ggirgin@hacettepe.edu.tr (G.G.); Tel.: +90-312-305-2178 \\ + Presented at the 2nd International Conference on Natural Products for Cancer Prevention and Therapy, \\ Kayseri, Turkey, 8-11 November 2017.
}

Published: 16 November 2017

\begin{abstract}
Oxidative stress is related to many pathologies and diseases including diabetes, atherosclerosis and cancer. Increase or decrease of reactive oxygen species can affect disease progression and treatment. Hence, both the development and treatment of diseases involve reactive oxygen species and antioxidants. Especially in the last decade, antioxidants became popular in the prevention of diseases and aging. Antioxidants exert their effects either by chain breaking or prevention of free radicals. In this study, the radical scavenging capacities and cytotoxic potentials of 5 non-enzymatic antioxidants were evaluated by in vitro analysis. The scavenging activities of ascorbic acid, glutathione, L-cysteine, $\mathrm{N}$-acetyl-cysteine and uric acid against $\mathrm{NO}, \mathrm{SO}, \mathrm{DPPH}$ radicals were analyzed. MTT and LDH assays were performed in order to evaluate the cytotoxic activities of the compounds in HeLa, HepG2 and Hep2 cell lines. All the compounds showed comparable antioxidant activities. On the other hand, cytotoxic effect of the compound was not significant except at high concentrations. The results was approved ones again all the compounds have strong radical scavenging activity and all the antioxidants may use for treatment of inflammatory diseases.
\end{abstract}

Keywords: ascorbic acid; glutathione; cysteine; uric acid; antioxidant

C 2017 by the authors. Licensee MDPI, Basel, Switzerland. This article is an open access article distributed under the terms and conditions of the Creative Commons Attribution (CC BY) license (http://creativecommons.org/licenses/by/4.0/). 